San Jose State University

SJSU ScholarWorks

$5-1-2006$

\title{
Cultural Identity of Labor and Delivery Nurses In the Assessment of Pregnant Patients For Interpersonal Violence
}

Ramona Nichols Smith

San Jose State University

Follow this and additional works at: https://scholarworks.sjsu.edu/etd_projects

Part of the Maternal, Child Health and Neonatal Nursing Commons

\section{Recommended Citation}

Smith, Ramona Nichols, "Cultural Identity of Labor and Delivery Nurses In the Assessment of Pregnant Patients For Interpersonal Violence" (2006). Master's Projects. 817.

DOI: https://doi.org/10.31979/etd.mjvv-zj92

https://scholarworks.sjsu.edu/etd_projects/817

This Master's Project is brought to you for free and open access by the Master's Theses and Graduate Research at SJSU ScholarWorks. It has been accepted for inclusion in Master's Projects by an authorized administrator of SJSU ScholarWorks. For more information, please contact scholarworks@sjsu.edu. 


\section{SAN JOSE STATE UNIVERSITY \\ SCHOOL OF NURSING}

\section{MASTER'S PROGRAM PROJECT OPTION (PLAN B) PROJECT SIGNATURE FORM}

STUDENT NAME: $\quad$ RAMONA NICHOLS SMITH

SEMESTER ENROLLED: $\quad$ SPRING 2006

TITLE OF PROJECT: CULTURAL IDENTITY OF THE LABOR AND

DELIVERY NURSE IN THE ASSESSMENT OF PREGNANT

PATIENTS FOR INTERPERSONAL VIOLENCE

NAME OF JOURNAL: Joumal of Obstetric, Gynecological, and Neonatal Nurse (JOGNN)

The project and manuscript have been successfully completed and meet the standards of the School of Nursing at San Jose State University. The project demonstrates the application of professional knowledge, clinical expertise, and scholarly thinking. An abstract of the project and two copies of the manuscript are attached.
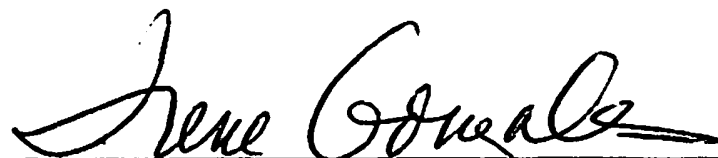

ADVISOR'S SIGNATURE

DATE

$\frac{\text { Dn. Bauman Wiluce }}{\text { ADVISOR'S SIGNATURE }} \frac{5 / 17 / 06}{\text { DATE }}$

Please submit this form to the Graduate Coordinator. Attach abstract, two copies of the manuscript, and documentation of submission to the-journal (i.e., Postal receipt) 
Submissions Being Processed for Author Ramona Nichols Smith, M... http://www.editorialmanager.com/jognn/auth_pendSubmissions.asp?...

Submissions Being Processed for Author Ramona Nichols Smith, MSN (05/06)

Page: 1 of 1 ( 1 total submissions)

Display 10 results per page.

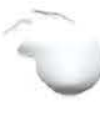

\begin{tabular}{|c|c|c|}
\hline Action & $\begin{array}{l}\text { Manuscript } \\
\text { Number }\end{array}$ & Title \\
\hline View Submis & & $\begin{array}{l}\text { Cultural Identity of Labor and Delivery Nurses in the Assessment of } \\
\text { Pregnant Patients for Interpersonal Violence }\end{array}$ \\
\hline
\end{tabular}

Initial Date

Submitted

$05 / 25 / 2006$ Pregnant Patients for Interpersonal Violence

Page: 1 of 1 ( 1 total submissions)
Status

Date

05/25/2006 $\begin{aligned} & \text { Submitted to } \\ & \text { Journal }\end{aligned}$

Display 10

results per page. 
Cultural Identity of Labor and Delivery Nurses

In the Assessment of Pregnant Patients

For Interpersonal Violence

Ramona Nichols Smith BSN, MS/NP Candidate

Irene Gonzales, $\mathrm{RN} \mathrm{PhD} \mathrm{CNP}$

Barbara Willard, RN DNP 


\author{
Author Identification Notes \\ Ramona Nichols Smith RN, BSN, MS/NP Candidate \\ Santa Clara Valley Medical Center, San Jose, CA \\ San Jose State University, San Jose, CA \\ Irene Gonzales PhD RN CNP \\ Associate Professor, FNP Program Director \\ San Jose State University, San Jose, CA \\ Barbara Willard DNP RN \\ Assistant Professor \\ San Jose State University, San Jose, CA
}

Acknowledgements

I wish to acknowledge Santa Clara Valley Medical Center, Watsonville

Community Hospital; and Chia-Ling Mao, $\mathrm{PhD}$, Associate Professor, San Jose

State University for their support. 


\begin{abstract}
Objective: Identification of barriers to assessment of interpersonal violence (IPV) in pregnant women.

Design: An exploratory descriptive study

Setting: The labor and delivery department of a public county hospital Participants: 34 nurses, representing 8 cultures and 13 native languages, completed the survey, and 34 laboring patient's medical records were reviewed. Main Outcome Measures: Any specific barriers, identified by nurses, to assessing for IPV in laboring patients

Results: Medical record review revealed $50 \%$ assessment rate in labor triage patients. Survey results revealed that cultural identity ( $85 \%)$ was not a significant barrier. Approximately $65 \%$ of nurses agreed that in their culture it was acceptable to ask patients about IPV. Over $88 \%$ of nurses stated their culture strongly supported asking about IPV. Over $50 \%$ of nurses identified language as the single most prevalent barrier in both US and non-US born nurses.
\end{abstract}

Conclusion: Labor nurse's cultural identity, in itself, was not a barrier to the assessment for IPV. A nurse's inability to speak the same language as the patient emerged as the single most significant barrier in the assessment for IPV in this study.

Keywords: interpersonal violence-IPV, cultural identity, abuse in pregnancy, assessment barriers 


\section{Callouts (3)}

1. Inability to communicate in the patient's language emerged as the most significant barrier for labor nurses, whether US or non-US born. (should appear in barriers to assessment)

2. To increase screening of pregnant women for IPV, we must understand the influence of language as a primary barrier. (should appear in discussion)

3. Conflict of languages presents a quality of care challenge as nurses are imported to fill staff shortages and increased immigration of non-English speaking patients continues. (should appear in implications for practice) 
Interpersonal violence (IPV) directed at women is epidemic world wide.

2 In the United States, we commonly hear the term, Domestic Violence (DV), in

3 describing the destructive effects on women and their children. Yet, the definition

4 of DV limits the abuser to an intimate partner. Often the abuser is not the father or

5 the current partner, but may be any other person(s) in the mother's life. This study

6 on interpersonal violence, did not limit data to a specific circumstance of abuse,

7 therefore included those situations defined within "Domestic Violence".

8 Women who are pregnant have an increased risk of becoming victims of

9 violence. AWHONN has supported routine education for nurses in the

10 identification and treatment for IPV (Schoening, Greenwood, McNichols,

11 Heermann, and Agrawal, 2004). It is known that abuse of women and children is

12 as clearly linked to alcohol abuse as are major motor vehicle accidents. The rate

13 of abuse rises 15 times higher in household where husbands are often drunk than

14 homes where the husband does not drink (Health and Healthcare 2010, 2003).

15 It is estimated that between $9 \%$ and $25 \%$ of pregnant women are abused

16 (Giardino, 1999; Cox 2003). Using the most modest estimate of only 4-5\%,

17 interpersonal violence in the prenatal period remains more common than diabetes

18 and preeclampsia, which are routinely screened for during pregnancy (Parsons,

19 Goodwin \& Petersen, 2000). Outcomes of pregnancies affected by IPV include

20 complications of first and second trimester bleeding, miscarriage, preterm labor, 
21 low birth weight infants, substance abuse, sexually transmitted diseases, and

22 urinary tract infections (McGrath, Hogan \& Peipert, 1998; Cox, 2003).

Upon admission to a labor and delivery service, assessing pregnant

24 patients for risk factors is clearly defined and standardized. This assessment

25 consistently includes screening for possible IPV.

26 Barriers to assessment for IPV include lack of formal training, lack of

27 privacy, feeling of helplessness to change the situation, and the personal belief

28 system of the specific nurse. Ellis (1999) reported lack of privacy and time

29 constraints as primary barriers in 40 RN's in a large trauma center. Additional

30 studies have reported a rate of IPV in the personal experience of nurses to be as

31 high as 58\% (Ellis, 1999; Cox, 2003; Denham, 2003).

Conceptual Framework

\section{Theory Description}

34 The Theory of Planned Behavior (TPB) provided the framework for this 35 study (Ajzen \& Fishbein, 1980). The central factor in TPB is the intention to

36 perform an identified behavior. In truth, the theory does not address the actual

37 control a person has, but the perceived behavior control. Though a person may be

38 willing to perform a certain behavior, realistic barriers may exist in their

39 perception of the ability to do so.

Application of the TPB to assessment for interpersonal violence would

41 have individual nurses show intent to screen when they approached it confidently, 
42 felt that others, important to them, thought they should do so, and believed the act

43 of intervention was under their control.

44 In the actual interaction of asking any woman about her safety and well-

45 being, there could have been a perceived ease or difficulty of performing the action. Research on what impacted the ease or the difficulty would aid in the

47 future goal of increasing compliance with laws and policy

When looking at health care, behavioral intention was the willingness on

the part of the nurse to perform a specific behavior; how much they were willing

to try to do it. This intention to perform the behavior or the action was rooted in

51 the attitude, subjective norms, and perceived behavioral control (Ajzen, 1988).

52 Therefore, it followed that if a willing intention could be provoked, then action of

53 the desired behavior would follow. The intervention that would affect one nurse's

54 behavior would not necessarily trigger action in another.

In this study, the desired behavior was the act of the nurse asking

questions of the pregnant patient regarding past or current interpersonal violence.

57 Without the core concepts resulting in the intention to perform the assessment,

58 there would be little hope of success. Yet even with the intention, the individual nurse needed to make the concerted effort to perform the act. 
Barriers to Assessment

Despite the knowledge of the need for assessment of the pregnant woman

66 for violence, assessments are missed. The multiple barriers have been identified in

67 the literature, yet ability to speak of the language of the patient has not been

68 identified to date as a significant factor (Ellis, 1999; Thompson, Rivara,

69 Thompson, Barlow, Sugg, and Maiuro, 2000). In a major review of 24 studies

70 that examined health care provider barriers, lack of time and lack of training were

71 the most often cited barriers (Parsons, Goodwin \& Petersen, 2000).

72

73

74

75

76

77

78

79

80

81 violence. Yet, conversely, the nurse may actually be a stronger advocate for the

82 patient due to this cultural experience. 
Training and Success Parsons, Goodwin and Petersen reported in 2000 that staff attendance at didactic training programs alone did not change screening behavior for the long term. In fact, training programs that combined instruction with institutional supports, such as a violence resource nurse, had greater success (Parsons, Goodwin, and Petersen, 2000). Certainly a referral to a nurse who speaks the same primary language as the client was essential.

\section{Clinical Decision Making}

Bakalis and Watson (2005) studied the clinical decisions nurses made in specific health care settings. No decision-making theories were applied. The aim of the study was to determine if decision-making varied based on the specialty of the nurse practice area. In conclusion, the authors posed an interest in knowing if nurses showed particular aptitudes for the different levels/or types of decisionmaking. Additionally, did the personality, education, or experience in nursing have any influence? Culture of origin of the nurse was not discussed or referenced in this study of 60 registered nurses (Bakalis \& Watson, 2005).

Method

\section{Research Design}

An exploratory, descriptive study was used to measure the self assigned cultural identity of labor and deliver nurses and the perceived barriers to assessing for interpersonal violence in their patients. Training had been provided and 
105 mandatory requirements for screening all women admitted to the triage area of

106 labor and delivery is well known. This descriptive study provided no treatment or 107 manipulation.

108 A literature search did not reveal a tool for assessment of the performance

109 of the mandatory screening with regard for the cultural identity of the nurse. An

110 instrument was developed specifically for this study by the Principal Investigator.

111 Demographics gathered the cultural factors of the participants, as well as the

112 perceived barriers to assessment for IPV.

113 The Smith Multicultural Questionnaire (SMQ) attempted to elicit

114 information about how cultural identity might influence the intent to assess for

115 IPV. The questionnaire was designed to inquire into three areas of influence.

116 First, how did the nurse's attitude, beliefs, and perceived outcomes influence the

117 intent to assess for IPV? Secondly, in what way did the influence of subjective

118 norms, or the social pressure to ask or not ask questions, influence clinical

119 decision-making about IPV? Thirdly, how did the perceived behavior control, or

120 the perception of the ease or difficulty of asking questions about IPV, influence

121 intent to assess?

122 The instrument was reviewed by two doctoral nursing faculty members at

123 San Jose State University, San Jose, CA; and three doctoral candidates from

124 University of California, San Francisco, CA for analysis of structure, validity, and

125 themes. Changes were made upon recommendations of the faculty. A pilot study 
126 was completed using labor and delivery nurses at a Community Hospital located

127 northern California.

128 Participants

129 The participants represented a self-selected sub sample from a

130 convenience sample comprised of 75 labor and delivery nurses. All participants

131 were registered nurses, participation was voluntary, and no incentives were

132 provided. Thirty four nurses completed the SMQ, which represented $45 \%$ of the

133 pool. Age of nurses ranged from 21 to 60 years of age (mean range $41-45 \pm 1.8$ )

134 (see Figure 1). The majority of the nurses had completed their baccalaureate

135 degree; had between eleven to fifteen years of registered nursing experience; and

136 were predominantly U. S. born (see Figures 2, 3, and 4).

137 Setting and Sample

138 The study was conducted in a busy labor and delivery department of a

139 524-bed public hospital owned and operated by a county in Northern California.

140 The total number of labor and delivery patient triage assessments in 2005 was

14111,203 . Of this number, 5887 were admitted for care and 5560 delivered their

142 pregnancy or a rate of approximately 463 births per month. The patients were

143 given prenatal care at 23 separate clinic sites who deliver at the study hospital.

144 Patients were primarily of Hispanic descent (74\%) and most were

145 monolingual Spanish Speaking. The next largest group was Caucasian women at

$14613 \%$. The remaining patients were Black (African or African American) 5\%, 
147 Asian 2\%, Filipino 1\%, Arab 1\%, Vietnamese 1\%, Indochinese .23\%, Pacific

148 Islander .16\% American Indian .05\%, and other or unknown $4 \%$.

149 The 75 nurses in labor and delivery represented twelve cultural identity

150 groups. The eight cultural identity groups represented by the 34 voluntary

151 participants (45 \%) included: Caucasian, Chinese, East Indian, Egyptian, Filipino,

152 Korean, Latina-Hispanic, and Nigerian (see Figure 4).

153 Measures

154 Nurse cultural identity was determined within the SMQ by direct question

155 "What culture do you identify with?" Twelve options and "other" were possible

156 responses. Place of birth did not necessarily indicate the nurses' individual sense

157 of her culture. Although several participants stated they were born in Canada, two

158 claimed "Caucasian" as their culture and not Canadian.

159 Barriers from the literature were introduced and reflected in the study

160 survey. Options that would represent family and culture as a barrier were added.

161 Cultural barriers included language spoken and family and/or cultural approval of 162 asking personal questions about relationships. The participants chose the one most

163 important barrier; and then any others that applied.

164 Research Procedure

165 Approval from two review boards (IRB) for the protection of human

166 subjects was obtained. Over a 4 week period of time, each nurse who agreed to

167 participate completed a consent form and a Smith Multicultural Questionnaire. 
168 The survey did not contain any identifying information to ensure the anonymity of 169 the participants.

170 A 24 hour/one day data collection of the triage intake forms from patient

171 medical record was conducted from the same study institution. The goal was to

172 detect the percentage of forms that included, or did not include, the required

173 assessment for IPV with the quality care standard set at $100 \%$.

174 The SMQ tool was introduced to the labor and delivery registered nursing

175 staff. Any qualified nurse participated by completing a consent and a survey. No

176 compensation was given for voluntary participation and the data remained

177 anonymous. All surveys were shredded following data collection by an

178 independent statistician.

Results

180 Medical Record Review

181 Thirty six admissions to labor and delivery triage took place within the

182 target 24 hours. Two were seen twice, giving the total number of patients at 34 .

183 Of the $34,(n=18)$ or $50 \%$ were asked about current or past interpersonal violence:

184 all responses were "no", as evidenced by notation in the medical record.

185 Gestational age of the pregnancy on admission was from 15 weeks (motor vehicle

186 accident) to $41+2$ weeks. Additional, only $50 \%$ of the 34 patients had

187 documentation of screening for IPV during prenatal care. 
189 findings for IPV were documented in $33 \%(n=6)$ of the patients who were actually

190 screened $(\mathrm{n}=18)$ in the prenatal period. The languages spoken by the patients with

191 IPV history were: English, Spanish, limited English, Spanish only, and Korean

192 only. The nurse participants spoke a total of 13 languages (see Table 1).

193 Barriers to Assessment

194 The initial assumption that a nurse's cultural identity would somehow be a

195 barrier was disproved. Interestingly, $85 \%$ of the participants stated that they

196 disagree that their culture would not approve of the nurse asking questions about

197 IPV with a significant level of $p=.013$. Only $9 \%(n=3)$ agreed that their culture

198 would not support their asking the IPV questions with one participant US born

199 and two were non-US born.

200 Additionally, $65 \%$ of the nurses agreed that in their culture it was

201 acceptable to ask questions about IPV. As for family approval of the nurses

202 asking the questions, $88 \%$ felt supported to do so.

203 Barriers to assessment were evaluated from the perspective of US

204 born and non-US born nurses. Both groups reported that inability to speak the

205 patient's language was the primary barrier for both US and non-US born nurses.

206 Inability to speak the patient's language was reported as the primary barrier by

$20750 \%$ of nurses with current or past abuse and $58 \%$ of the nurses without personal

208 experience of IPV. 
Clearly, when the language of the nurses varied from that of the patients;

210 barriers to care existed. A total of $80 \%$ of nurses reported language as a primary

211 or secondary barrier. Results of the current study departed from prior research in

212 that the issue of language was neither studied nor identified as a barrier to IPV

213 assessment.

The labor and delivery nurses self reported that only $73 \%$ screen routinely

216 for IPV in their patients, yet the actual documented assessment was only $50 \%$ in

217 the medical record review. These findings are consistent with the literature as

218 Ellis (1999) reported $45 \%$ of the nurses stated they routine screen all their patients 219 and only $9 \%$ of the charts reflected it was done. Alarmingly, with only half of the 220 patients being assessed in the prenatal period, the labor triage visit might have

221 been the only opportunity for intervention in several of the patients.

222 A nurse's inability to speak the same language as the patient emerged as

223 the most significant barrier in the assessment for IPV (see Table 2). Despite the

224 unique cultural environment at the study institution, we have begun to see this

225 trend of language barrier nationwide due to our importation of $\mathrm{RN}$ workforce and

226 increased immigration of non-English speaking patients,

\section{Limitations}

228 Limitations of the study include the small convenience sample size. The 229 unique cultural diversity of the nurses at the institution studied may not be similar 
230 to other labor and delivery departments in institutions of similar size. Yet, this

231 complex diversity of cultural identity may represent the future of nursing in the

232 United States.

233

234

235

236

237

238

239

240

241

242

243

244

245

246

247

248

249

250
Implications for Practice

Despite the multicultural diversity in the nursing staff studied, it did not match the client diversity in culture or language. The answer may not be in language education, but perhaps in the development of non-verbal tools similar to the Wong-Baker Pain Scale we use routinely for pain assessment. Provision of a screening tool for nurses, nurse practitioners and physicians would allow initial screening. Follow up with a translator in the event of positive findings would be indicated

Research is lacking in the area of identification and study of the impact of culture and language in the practice of nursing. Xu reports that the typical internationally educated nurses are recruited from the Philippines, Canada, India or the United Kingdom, yet language was not mentioned in the article on the economics of dealing with the nursing shortage (Xu, 2005).

Hospitals, whose patients speak different languages, are responsible to their patients by providing resources in the form of translators and translating systems. These resources are not standardized and are less than sufficient to meet patient needs. This study adds to the body of knowledge further showing how important it to have nurses available who can speak different languages, 
251 especially the primary languages spoken by the local patient community. This

252 study further supports the premise of how inadequate and, yet vital, language

253 translation resources are for the safety and optimal care of patients. 


\section{References}

Ajzen, I. (1988). Attitudes, personality, and behavior. Chicago, IL: Dorsey Press.

Ajzen, I., \& Fishbein, M. (1980). Understanding attitudes and predicting social behavior. Englewood Cliffs, NJ: Prentice-Hall.

Bakalis, N., \& Watson, R. (2005). Nurses' decision-making and clinical practice. (art and science research). Nursing Standard, 19, 33-40.

Cox, E. (2003). Synergy in practice: Caring for victims of intimate partner violence. Critical Care Nursing Quarterly, 26, 323-330.

Denham, S. (2003). Describing abuse of pregnant women and their healthcare workers in rural appalachia. The American Journal of Maternal/Child Nursing, 28, 264-269.

Doll, J. \& Ajzen, I. (1992). Accessibility and stability of predictors in the theory of planned behavior. Journal of Personality and Social Psychology, 63, 754-765.

Giardino, E. R. (1999). Uncovering abuse in the pregnant woman. Nursing Spectrum, 9, 11-14.

The Institute for the Future. (2003). Health and health care 2010; the forecast, the challenge. Princeton: Jossey-Bass.

Khosla, A., Dua, D., Devi, L., \& Sud, S. (2005). Domestic violence in pregnancy in north Indian women. Indian Journal of Medical 
Sciences, 59, n/a. Retrieved October 13, 2005 from Medknow Publications, Electronic Collection: A133850715.

McGrath, M.E., Hogan, J.W., \& Peipert, J.F. (1998). Prevalence survey of abuse and screening of abuse in urgent care patients. Obstetrics \& Gynecology, 91, 511-514.

Parsons, L., Goodwin, M.M., \& Petersen, R. (2000). Violence against women and reproductive health: toward defining a role for reproductive health care services. Maternal and Child Health Journal, 4, 135-140.

Schoening, A., Greenwood, J, McNichols, J. Heermann, J. \& Agrawal, S. (2004). Effect of an intimate partner violence education program on the attitudes of nurses. Journal of Obstetrical, Gynecologic, and Neonatal Nursing, 33, $572-579$.

Thompson, R. S., Rivara, F.P., Thompson, D. C., Barlow, W. E., Sugg, N. K. Maiuro, R. D., et al. (2000). Identification and management of domestic violence: A randomized trial. American Journal of Preventive Medicine, 19, 253-263.

Xu, Y., Kwak, C. (2005). Characteristics of internationally educated nurses in the United States. Nursing Economics, 23, 233-238. 


\section{Age of Nurses (years)}

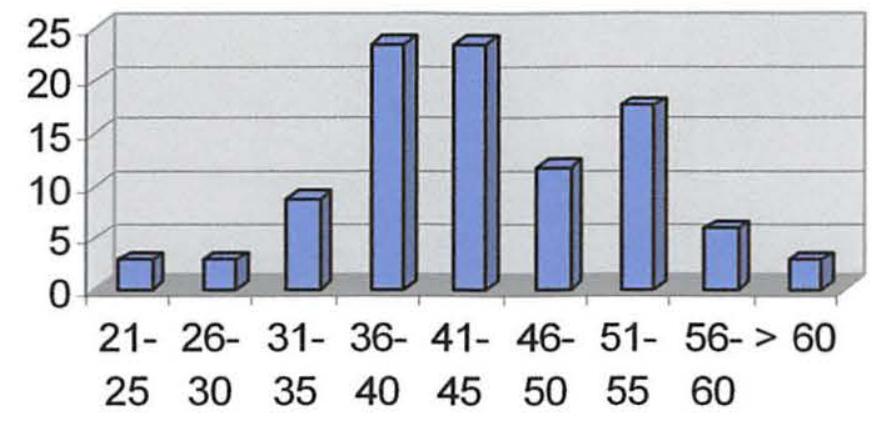

Figure 1. Characteristics of Nurse Participants: Age $(\mathrm{n}=34)$ 


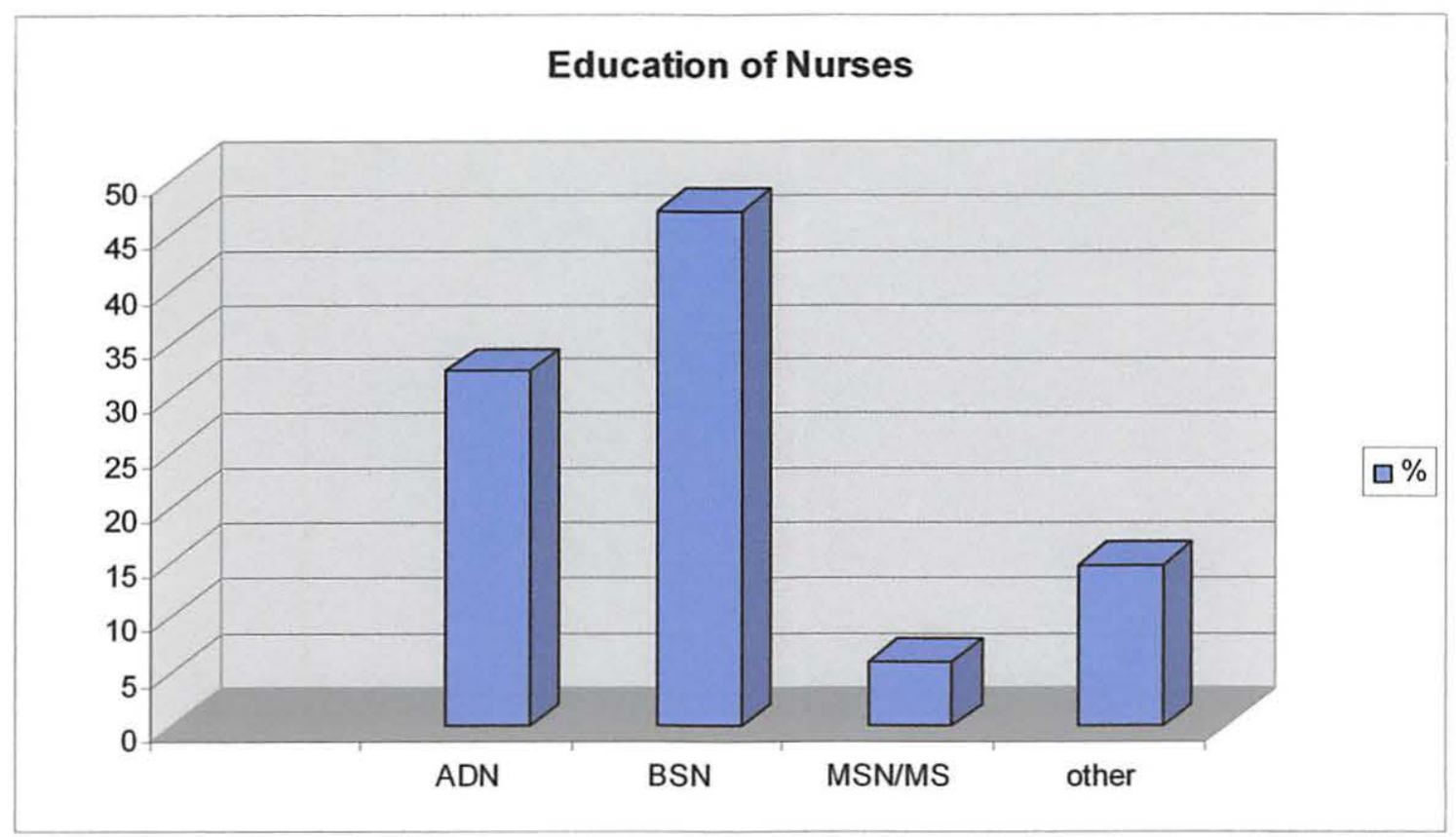

Figure 2. Characteristics of Nurse Participants: Education $(n=34)$

$\mathrm{ADN}=$ Associate degree in nursing

$\mathrm{BSN}=$ Baccalaureate degree in nursing

MSN/MS = Master's degree in nursing or other related field

Other $=$ Non-nursing associate and baccalaureate degree 


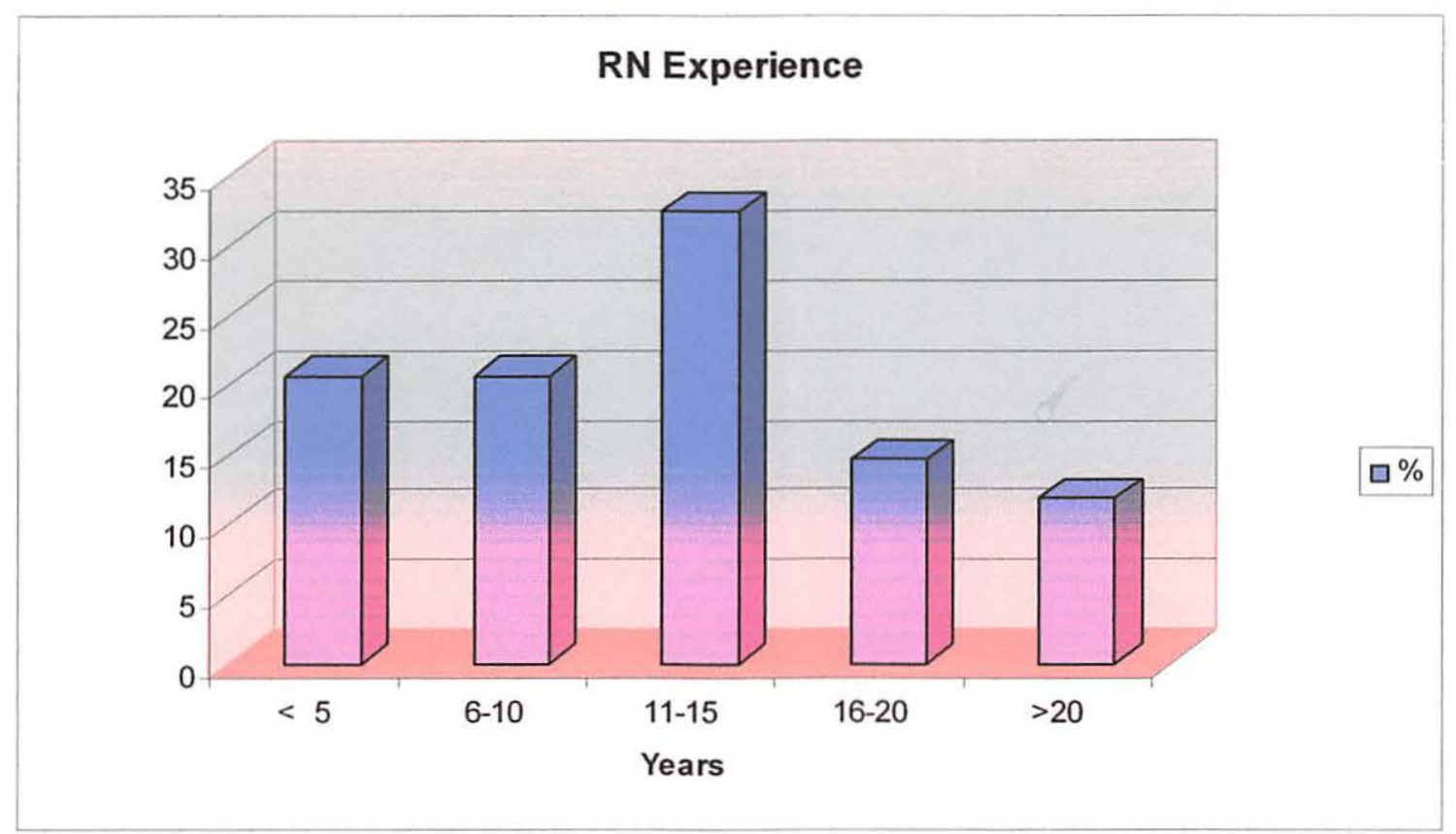

Figure 3. Characteristics of Nurse Participants: Years of Experience $(\mathrm{n}=34)$ 


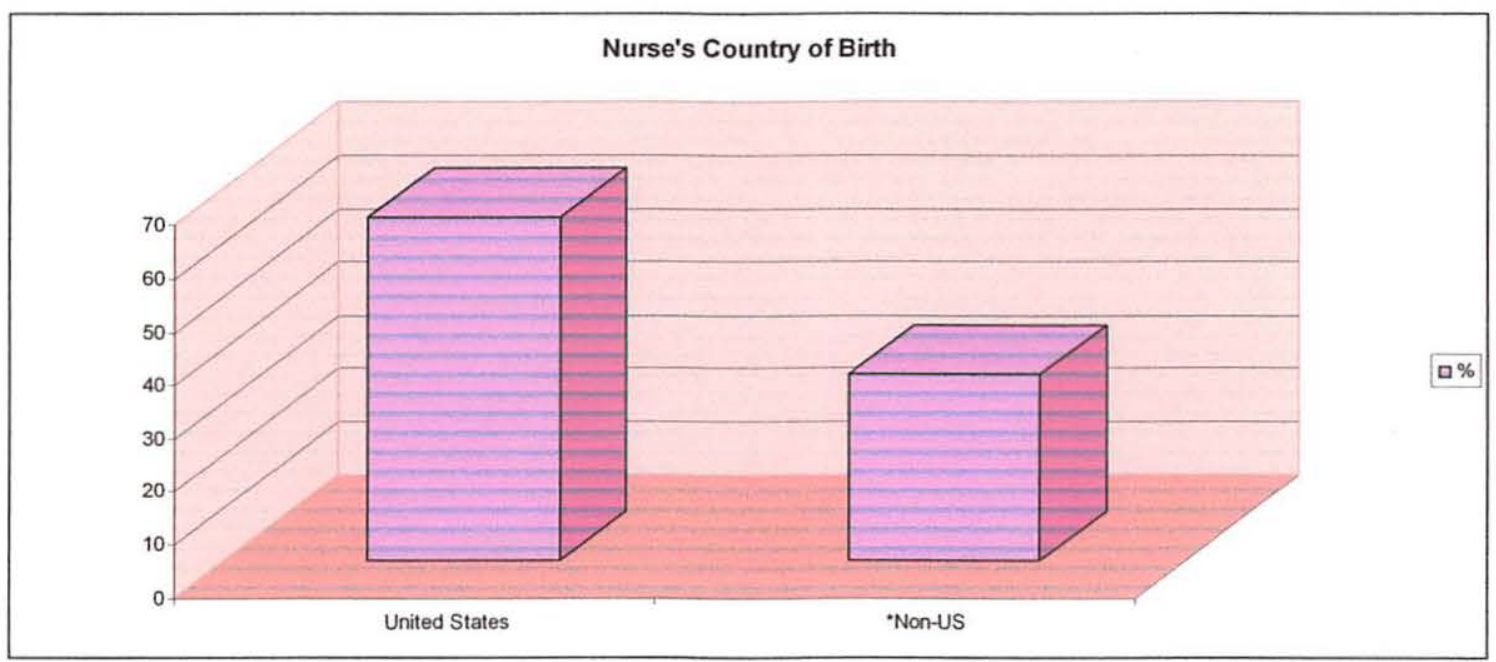

Figure 4. Characteristics of Nurse Participants: Nurse's Country of Birth $(n=34)$ *Cambodia, Canada, Chile, Egypt, India, Korea, Nigeria, Philippines 
Table 1

Comparisons of Nurse's and Patient's Language

\begin{tabular}{|c|c|c|c|c|}
\hline \multirow[b]{2}{*}{ Language Spoken } & \multicolumn{2}{|c|}{ Nurses $(n=34)$} & \multicolumn{2}{|c|}{ Patients $(n=34)$} \\
\hline & $\mathrm{n}$ & $\%$ & $\mathbf{n}$ & $\%$ \\
\hline English & 34 & 100 & 6 & 17.6 \\
\hline Spanish + English & 16 & 47 & 4 & 11.8 \\
\hline Spanish only & 0 & 0 & 22 & 64.7 \\
\hline Korean + English & 1 & 2.9 & 0 & 0 \\
\hline Korean only & 0 & 0 & 1 & 12.9 \\
\hline Arabic & 1 & 2.9 & 0 & 0 \\
\hline *Bini & 1 & 2.9 & 0 & 0 \\
\hline Chinese & 1 & 2.9 & 0 & 0 \\
\hline Filipino/Tagalog & 2 & 5.9 & 1 & 2.9 \\
\hline French & 4 & 11.8 & 0 & 0 \\
\hline Hindi & 1 & 2.9 & 0 & 0 \\
\hline **Igbo & 1 & 12.9 & 0 & 0 \\
\hline †Punjabi & 2 & 5.9 & 0 & 0 \\
\hline **Yoruba & 2 & 5.9 & 0 & 0 \\
\hline Vietnamese & 1 & 12.9 & 0 & 0 \\
\hline
\end{tabular}


Table 2

Barriers to Screening for Interpersonal Violence Identified by Nurses $(n=34)$

\begin{tabular}{|c|c|c|c|c|}
\hline \multirow{3}{*}{\begin{tabular}{|l|} 
Barrier Description (based on survey questions) \\
\end{tabular}} & \multicolumn{2}{|c|}{ 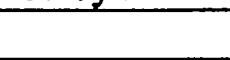 } & \multirow{2}{*}{\multicolumn{2}{|c|}{ Additional }} \\
\hline & & & & \\
\hline & $\mathbf{n}$ & $\%$ & $\mathrm{~N}$ & $\%$ \\
\hline 1. Area on form not conveniently located & 0 & 0 & 3 & 8.8 \\
\hline 2. I don't feel it is really my job to screen & 0 & 0 & 3 & 8.8 \\
\hline $\begin{array}{l}\text { 3. There is lack of privacy for screening in my heath } \\
\text { care setting }\end{array}$ & 10 & 29.4 & 10 & 29.4 \\
\hline 4. I don't know what to do if the answer if yes & 1 & 2.9 & 5 & 14.7 \\
\hline $\begin{array}{l}\text { 5. I don't feel I have the support from nursing } \\
\text { management }\end{array}$ & 0 & 0 & 1 & 2.9 \\
\hline $\begin{array}{l}\text { 6. I do not speak the patient's language well enough } \\
\text { to ask sensitive questions }\end{array}$ & 18 & 52.9 & 9 & 26.5 \\
\hline 7. I feel the patient will stay with the abuser anyway & 0 & 0 & 4 & 11.8 \\
\hline 8. I feel uncomfortable asking the questions & 0 & 0 & 2 & 5.9 \\
\hline $\begin{array}{l}\text { 9. A woman must try to deal privately with abuse in } \\
\text { her own way }\end{array}$ & 0 & 0 & 3 & 8.8 \\
\hline $\begin{array}{l}\text { 10. I don't know enough about the issues of } \\
\text { interpersonal violence to assess for it }\end{array}$ & 0 & 0 & 1 & 2.9 \\
\hline 11. I cannot fix the problem anyway & 1 & 2.9 & 2 & 5.9 \\
\hline $\begin{array}{l}\text { 12. I have been abused and do not feel can bring up } \\
\text { the issue with my patients }\end{array}$ & 0 & 0 & 2 & 5.9 \\
\hline *13. I don't feel I have support from my colleagues & 0 & 0 & 0 & 0 \\
\hline $\begin{array}{l}\text { 14. In my culture it is not acceptable to ask about the } \\
\text { relationships in this way }\end{array}$ & 1 & 2.9 & 0 & 0 \\
\hline 15. I do not have adequate trainir & 3 & 8.8 & 3 & 8.8 \\
\hline
\end{tabular}

Note. *Based on data, nurses do think they have support from colleagues. 